\title{
Tunable electrophysical properties of composites nano-CdS/polyvinyl alcohol
}

\author{
G.Yu. Rudko a , A.O. Kovalchuk ${ }^{a,{ }^{*}}$, V.A. Bondarenko a , V.I. Fediv ${ }^{\mathrm{b}}$, E.G. Gule ${ }^{\mathrm{a}}$ \\ ${ }^{a}$ V. Lashkaryov Institute of Semiconductor Physics of National Academy of Sciences of Ukraine, 45, Pr. Nauky, Kiev 03028, Ukraine \\ ${ }^{\mathrm{b}}$ Department of Biophysics and Medical Informatics, Bukovinian State Medical University, 42 Kobylyanska St., 58000 Chernivtsi, Ukraine
}

\section{H I G H L I G H T S}

- Electrical d.c. conductivity of nano-CdS/polyvinyl alcohol composites are studied.

- Temperature dependence of d.c. conductivity of composites are studied.

- Influence of nanoparticles concentrations on d.c. conductivity were investigated.

- Current-voltage characteristics interpreted in term of space-charges-limited currents.

- Tunability of the thermal sensitivity of CdS/polyvinyl alcohol composite is analyzed.

\section{A R T I C L E I N F O}

\section{Article history:}

Received 17 November 2013

Received in revised form

24 May 2014

Accepted 11 July 2014

Available online 1 August 2014

\section{Keywords:}

Polymers

Nanostructures

Composite materials

Electrical conductivity

\begin{abstract}
A B S T R A C T
$\overline{\text { Electrical d.c. conductivity of nano-CdS/polyvinyl alcohol composites with different nanoparticle con- }}$ centrations and its temperature dependence were studied. The composites demonstrate increasing resistivity with CdS nanoparticles content. The current-voltage characteristics of composites exhibit non-ohmic superlinear behavior that is interpreted in terms of space-charges-limited currents. Temperature dependence of resistivity is characterized by high temperature coefficient of resistivity which is favorable for applications in flexible, easy-to-shape thermistors. The tunability of the thermal sensitivity of CdS/polyvinyl alcohol composites was analyzed.
\end{abstract}

() 2014 Elsevier B.V. All rights reserved.

\section{Introduction}

Control of the electrical conductivity of polymers by mixing them with various salts has attracted a lot of attention and has been addressed in many papers [1-4]. With the development of nanotechnology, new methods have appeared for modifying the properties of widely used polymers by adding semiconducting and metallic nanoparticles (NPs) [5-7]. Due to quick progress in this field, polymer-based nanocomposites are considered prospective materials for various applications ranging from sensors to solar cells, thus, the possibilities to control their properties are of great interest.

A nano-composite material is expected to exhibit the combined properties of its components or even enhanced properties as compared to its components [[8] and references therein]. For

\footnotetext{
* Corresponding author. Tel.: +38 (097)6694174.

E-mail address: andriy_kovalchuk@yahoo.com (A.O. Kovalchuk).
}

example, previous studies of the nano-CdS/polyvinyl alcohol composite have revealed new optical [9-12] and electrical [17,18] properties.

The electrical conductivity of polyvinyl alcohol (PVA) have been studied in a series of papers [2,4,14-16], however, there is no consensus in literature as to the nature of the conductivity in this polymer. A variety of conductivity mechanisms were proposed in order to describe the current flow in PVA, such as variable-range hopping [13], Shottky-type conductivity [2,4], and Poole-Frenkel mechanism [14,15].

Similarly, the diversity of results and interpretations occur in the field of nanocomposite research. Studies on the electrical properties of nanocomposites were mostly conducted on polymers loaded with metallic nanoparticles or doped with various salts [for doped PVA see, e.g. Refs. $[2,4,14,16]]$. Electrical properties of polymers loaded with semiconductor nanoparticles attracted much less attention; namely, the results for composites CdS/PVA were reported in Refs. [17,18]. The conclusions of these papers are to some extent contradictory. According to Ref. [18], the conductivity of the 
composite at low fields is linear and changes to quadratic current-voltage dependence at higher fields. This behavior is interpreted as the transition from the ohmic regime to the regime of the injection by space-charges-limited currents. In the paper [17] the PVA-based composites loaded with rather large nanoparticles (with diameters more than $700 \mathrm{~nm}$ ) were studied. Those composites have high electronic conductivity which increases linearly with an increase in concentration of CdS. The authors proposed the hopping mechanism of conductivity to interpret the data they obtained on dc conductivity as a function of concentration and temperature.

Here we present the results of the studies of the electrical properties of the nanocomposite comprising the polymeric matrix of polyvinyl alcohol (PVA) with the semiconductor CdS nanoparticles (NPs). We also analyze the mechanisms of dc conductivity and demonstrate the possibilities for varying the electrical properties of this composite by changing the polymer load.

\section{Experimental}

\subsection{Procedure of nanocomposite synthesis}

The nanocomposites CdS/PVA were obtained by synthesizing nanoparticles of CdS in a water solution of PVA that served as a capping agent to restrict the NPs growth and to prevent their coagulation. The degree of hydrolysis of the PVA used was about 99.0-99.5\%. The starting solution for growing NPs contained 5 (wt.)\% of PVA. It was obtained by dissolving polymer in double distilled deionized water at $90{ }^{\circ} \mathrm{C}$. All other synthesis steps were done under ambient conditions.

Analytical-grade $\mathrm{CdCl}_{2}$ and $\mathrm{Na}_{2} \mathrm{~S}$ salts were used as the precursors for growing NPs. The concentrations of salts in the growth solution as well as the $\mathrm{pH}$ value (which was equal to 5 ) were kept at levels that prevented the formation of $\mathrm{Cd}(\mathrm{OH})_{2}$. To keep the concentration of precursors constant while the NPs grew, the salts were gradually injected into the solution during the synthesis process. The growth procedure lasted for $\sim 2 \mathrm{~h}$. The final product of this multi-step synthesis procedure was a colloidal solution of CdS NPs with an average diameter of $5.4 \mathrm{~nm}$ (a typical transmission electron microscope image can be seen in Ref. [19], Fig. 1).

This product was further diluted by the same water solution of PVA that was used as a starting solution, and colloidal solutions with a NPs concentration of two- and four-times less were obtained.

To obtain thin solid films of composites for electrical measurements, equal portions of colloidal solutions with different concentrations of NPs were placed in glass Petri dishes and then were dried at room temperature in a closed vessel containing an absorbent. The same amount of pure PVA was also dried to obtain a reference sample of solid PVA film. The highest concentration of NPs in the film produced from the most concentrated colloid was $c_{0}=4^{*} 10^{16} \mathrm{~cm}^{-3}$. Hereafter we shall denote the samples in accordance with the increasing fraction of NPs: 0 - pure PVA, CdS/PVA 1 , CdS/PVA 2, and CdS/PVA 3 - the nanocomposites with $c_{0} / 4, c_{0} / 2$ and $c_{0}$ concentrations of NPs, respectively. The dried films were flexible, transparent, colorless (sample 0 ) or light-yellow of gradually increasing tone (samples $1-3$, respectively). The average thickness of all films was about $250 \mu \mathrm{m}$.

\subsection{Electrical measurements}

The dc resistance of the nanocomposite samples was measured along the film by the two-probe method at ambient conditions. An experimental cell was assembled using either slightly springloaded contacts of $\mathrm{Ag}-\mathrm{Cu}-\mathrm{Ni}$ alloy or silver paste contacts. The resistance of the contacts was at least by an order of magnitude lower than the resistance of the samples. This was checked both by four-probe measurements and by the variation of the distance between the contacts at two-probe measurements. To minimize the influence of near-contact regions on the conductivity of the sample as a whole, the length of the layer employed between electrodes was much larger than the contact sizes. Electrical conductivity was calculated taking into account the geometry of the samples.

Special attention was paid to provide stationary conditions all electrical measurements. It is known that under the applied electrical field the PVA, similarly to many other dielectric polymers, is polarized [20]. The polarization is due to the space charge formed by the carriers which were injected to the polymer from the electrodes and trapped in the bulk, dipolar orientation, migrational polarization, etc. As a result, after the application of voltage to the dielectric, the current flowing through the sample decays and stabilizes at a certain value. This relaxation takes a rather long time, approximately several minutes, similar to the observations reported in Refs. $[18,20]$. Taking into account PVA's known polarization behavior in an electric field, the resistance of the sample was measured after the stabilization of the current in the circuit.

To measure dc current-voltage characteristics, the experimental cell was connected in series to a variable voltage source and a $100 \mathrm{k} \Omega$ load resistor (this value is several orders of magnitude less than the sample's resistance). The applied voltage was varied in the range of 100-1000 V. To measure the current in the circuit, a V7-21 digital voltmeter was connected in parallel to the load resistor.

The temperature dependencies of conductivity were measured using an E6-13A teraohmmeter at $(100 \pm 10) \mathrm{V}$. The sample was mounted into a thermostatically controlled furnace, and the temperature was varied within a range of $20-60{ }^{\circ} \mathrm{C}$. Higher temperatures were avoided to prevent the glass transition in the polymer that occurs at $80-85{ }^{\circ} \mathrm{C}$. The temperature of the samples was controlled by a copper-constantan thermocouple with an accuracy of $\pm 1{ }^{\circ} \mathrm{C}$. The thermocouple was placed as close to the sample as possible (to minimize the effect of temperature gradient) but without direct contact with the sample.

\section{Experimental results}

We have studied conductivity, temperature dependencies of conductivity, and current-voltage characteristics of CdS/PVA nanocomposites with different contents of NPs. It was observed that an increase in the concentration of NPs in a composite leads to a decrease of conductivity (see Table 1 ).

The temperature dependencies of the resistance of nanocomposite samples (Fig. 1, a) demonstrate that, with an increase in the concentration of NPs, the response of the samples' resistance to the temperature variation is largely enhanced. To characterize this response, a temperature coefficient of resistance $\left(\alpha_{\mathrm{T}}\right)$ is commonly used: $\alpha_{\mathrm{T}}=1 / R(T) \cdot d R / d T\left[\mathrm{~K}^{-1}\right]$. We have observed that the value of $\alpha_{\mathrm{T}}$ at a fixed temperature increases with the concentration of the semiconductor phase (see Table 1 ).

Results are also presented in the form of a $\ln \left[\sigma_{\mathrm{dc}}(\mathrm{T})\right]$ vs. $1000 / T$ graph (Fig. 1b) in order to find the activation energy of conductivity. These plots demonstrate that there is only one slope for the unloaded PVA and two slopes for all other composite samples. The activation energy of conductivity for all the investigated samples has been calculated using the Arrhenius equation

$\sigma=\sigma_{0} \cdot \exp \left[(-E) /\left(K_{\mathrm{B}} T\right)\right]$

where $\sigma$ is the conductivity at temperature $T, \sigma_{0}$ is a constant, $E_{\mathrm{a}}$ is the activation energy, and $k_{\mathrm{B}}$ is the Boltzmann constant. The estimated activation energy for pure PVA is equal to $0.32 \mathrm{eV}$. Two slopes on the Arrhenius plots for the composite samples correspond to the activation energy values $0.32 \mathrm{eV}$ and $0.87 \mathrm{eV}$. These 

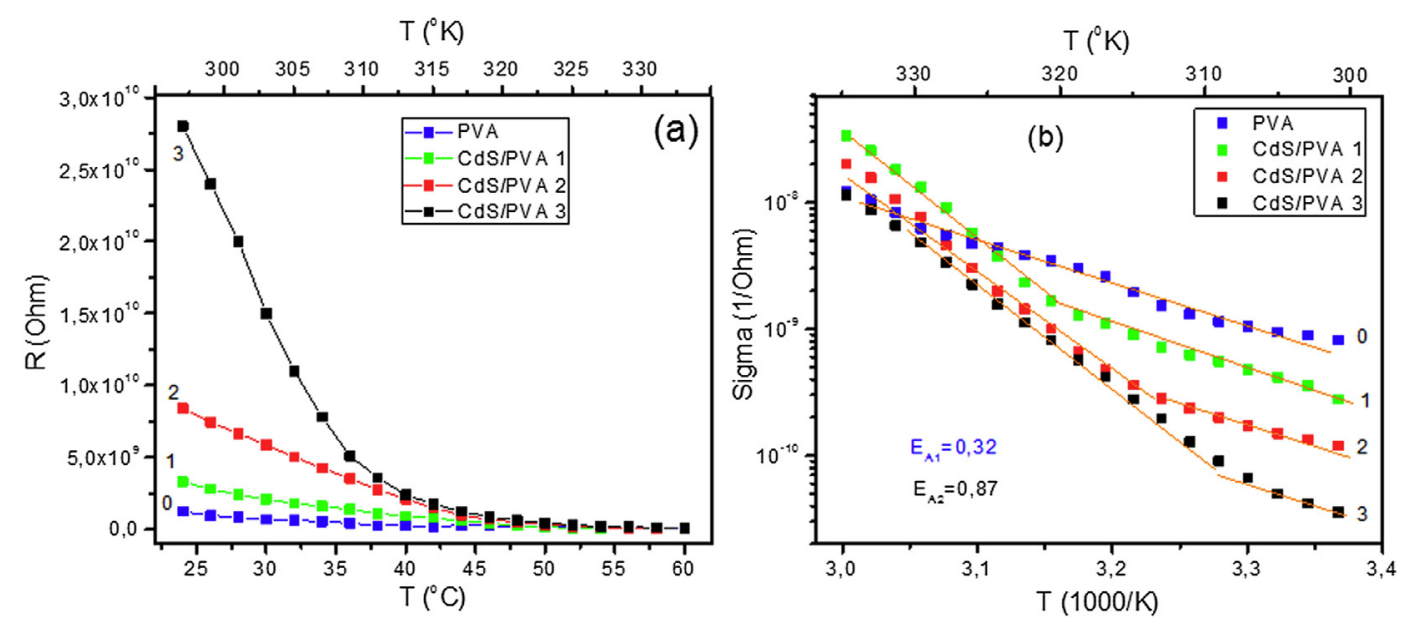

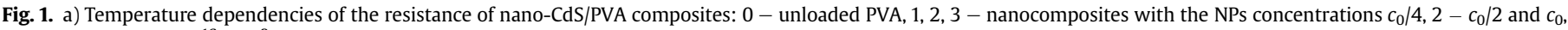
respectively. $c_{0}=4^{*} 10^{16} \mathrm{~cm}^{-3}$. b) Temperature dependencies of conductance presented in the Arrhenius coordinates.

values are the same for all composite samples regardless of the content of NPs (see also Table 1). It should be noted that a slope change is observed at different temperatures depending on the concentration of NPs: the higher concentration, the lower the temperature of the crossover to higher activation energy, and, correspondingly, to higher values of $\alpha_{\mathrm{T}}$.

The dependence of the current vs. voltage applied to the films of pure PVA and the composite samples with different concentrations of NPs are presented in Fig. 2 in double logarithmic coordinates. I-V characteristics of all samples demonstrate superlinear behavior, thus, the conductivity of all samples deviates from Ohm's law.

For all composite samples the current-voltage characteristics (curves 1-3 in Fig. 2) can be linearly fitted in the whole range of applied voltages. The value of the corresponding slope decreases with the increase in NPs content in a sequence of 2.9,2.7, and 2.5 for the samples 1,2 , and 3, respectively.

More complicated behavior is observed for pure PVA. Its current-voltage dependence shows two regions: in the low field range, the $I-V$ curve is linear in the double logarithmic scale with a slope of 3.1; while in the region of higher field values, the slope of the $I-V$ curve changes. Detailed analysis shows that in the high field range, the curve can be better fitted by a straight line in the Poole-Frenkel coordinates (see the insert to Fig. 2) which points to a change in the conductivity mechanism.

\section{Discussion}

\subsection{Conductivity altering by introducing nanoparticles into the polymeric matrix}

Keeping in mind that the polymeric matrix of CdS/PVA composite is a typical insulator, it is quite reasonable to suggest that the introduction of semiconductor inclusions (that have higher conductivity than PVA) would increase the conductivity of the system as a whole. This expected behavior was observed in Ref. [17] for the CdS/PVA composite. In Ref. [12] it was proposed that the conductivity in a composite occurs via the hopping of charge carriers between CdS NPs that are separated by insulating polymer and which act as a potential barrier to inter-nanoparticle hopping. Thus, an increase in the concentration of NPs would improve the conductivity by increasing the probability of hops.

Quite differently, our nanocomposites demonstrate higher resistivity than the unloaded polymer; introducing higher concentrations of NPs leads to a further increase in resistivity (see Table 1).
This apparent discrepancy in the conductivity behaviors of two similar materials can be caused by the difference in the structure of CdS/PVA composites studied here and in Ref. [17]. The most important distinction is the tremendous difference in the particle sizes: the CdS NPs studied in Ref. [17] were almost by two orders of magnitude larger than the sizes of NPs in our samples. Another factor is the difference in NPs content in the samples: the filling of our samples (which does not exceed $0.32 \%$ ) is much lower than the filling of composites (10-40\%) studied in Ref. [17]. Both factors point to the different roles of NPs in the conductivity processes in these composites. At low levels of filling and, as a result, at much larger distances between the NPs, the probability of hopping is strongly diminished. Consequently, the hopping mechanism of conductivity can be rejected. The decrease in conductivity with NPs content in our case implies that NPs play the role of the traps for the charge carriers available in polymer: the higher the NPs content (i.e., the more traps), the less free carriers available for participating in conductivity.

The reason why CdS NPs can trap carriers from PVA is because of the difference in the band gap values between the polymer and the NPs. The value of the HOMO-LUMO gap of PVA is $\sim 6.0 \mathrm{eV}$ and the band gap of CdS NPs with an average size of $5.4 \mathrm{~nm}$ is $\sim 2.6 \mathrm{eV}$ (the band alignment for the CdS/PVA system can be found in Fig. 4 in paper [19]). Thus, introducing NPs into the polymer matrix can produce rather deep traps for charge carriers. At moderate temperatures, the intrinsic carriers of the polymeric matrix must be localized by these traps. As a result, the concentration of the carriers in polymer diminishes that corresponds to decreasing of the sample conductivity. Another consequence of the presence of nanoparticle-related traps is that behavior differs with increasing temperature (to be discussed in Section 4.2).

\subsection{Conductivity mechanisms in unloaded PVA and composite}

In composite samples with low concentrations of NPs, the current flow through the sample occurs predominantly within the polymeric matrix. Thus, we started the analysis of conductivity mechanisms by examining the processes in PVA. At room temperature, PVA polymer is partly crystalline and partly amorphous. Thus, similarly to other disordered systems, the density of states has tails in the band gap of PVA. The states within the tails serve as traps for electrons. In what follows we will assume that the distribution of these traps is exponential as is typical for amorphous materials (the proposed scheme of electronic energy states for pure 
Table 1

Structural and electrical characteristics of polymer and composite samples.

\begin{tabular}{|c|c|c|c|c|c|c|c|c|}
\hline Sample & $\begin{array}{l}\text { Volume fraction of } \\
\text { the semi-conductor } \\
\text { phase, \% }\end{array}$ & $\begin{array}{l}\text { Nanoparticles } \\
\text { concentration, } \\
\mathrm{cm}^{-3}\end{array}$ & $\begin{array}{l}\text { Average distance } \\
\text { between the } \\
\text { nanoparticles, nm }\end{array}$ & $\begin{array}{l}\text { Conductivity, } \\
\Omega^{-1} \mathrm{~cm}^{-1} \\
\left(\text { at } 24{ }^{\circ} \mathrm{C} \text { ) }\right.\end{array}$ & $\begin{array}{l}\text { Temperature } \\
\text { coefficient of } \\
\text { resistance (at } 24{ }^{\circ} \mathrm{C} \text { ) }\end{array}$ & $\begin{array}{l}\text { Temperature } \\
\text { coefficient of } \\
\text { resistance (at } 37^{\circ} \mathrm{C} \text { ) }\end{array}$ & $\begin{array}{l}\text { Slope of the } \\
\ln (I)-\ln (V) \\
\text { plot }\end{array}$ & $\begin{array}{l}\text { Activation } \\
\text { energy of } \\
\text { conductivity, eV }\end{array}$ \\
\hline 0 & - & - & - & $3.3 \cdot 10^{-8}$ & 0.042 & 0.039 & $3.1^{\mathrm{a}}$ & 0.32 \\
\hline 1 & 0.08 & $10^{16}$ & 45 & $1.4 \cdot 10^{-8}$ & 0.042 & 0.105 & 2.9 & $0.32 ; 0.87$ \\
\hline 2 & 0.16 & $2 \cdot 10^{16}$ & 35 & $4.7 \cdot 10^{-9}$ & 0.042 & 0.105 & 2.7 & $0.32 ; 0.87$ \\
\hline 3 & 0.32 & $4 \cdot 10^{16}$ & 27 & $1.4 \cdot 10^{-9}$ & 0.042 & 0.105 & 2.5 & $0.32 ; 0.87$ \\
\hline
\end{tabular}

a In the range $100-400 \mathrm{~V}$.

PVA at zero and low field is shown in Fig. 3a). The scheme represents the exponential dependence of the density of trap states within the band gap vs. energy counted from the bottom of the conductivity band and the level $E_{\mathrm{d}}$ that corresponds to some donor states (the role of these states will be discussed below when the behavior at high field is analyzed). At equilibrium, the traps below the Fermi level are occupied by electrons (see the shaded area below $E_{\mathrm{F}}$ in Fig. 3a); whereas, other traps are empty and the intrinsic conductivity of the polymer is extremely low. Similarly, the electrons from level $E_{\mathrm{d}}$ have moved to the lower-lying trap levels in the tails of the density of states.

When a low field is applied, the electrons are injected into the dielectric from the electrode, partly filling the remaining empty traps, and the Fermi level slightly shifts to higher energies. The trapped carriers form a space charge that is distributed within the bulk of the sample. Thus, at low injection levels (in the low voltage region), the conductivity can be described by the model of spacecharge-limited current. The corresponding $I-V$ characteristics are expressed by the superlinear dependence $I \sim V^{n}$, where $n$ is the nonlinearity index. For the unloaded polymer, the index $n$ is equal to 3.1 in the range of $100-400 \mathrm{~V}$ (see Table 1 ). This observation correlates with the data from paper [14] where dependence $I \sim V^{2.75}$ was observed. The value $n>2$ is characteristic for a disordered solid with an exponential distribution of traps [21] and corroborates our assumption regarding the exponential-like tail of the density of states. In this low field range, the presence of the donor level $E_{\mathrm{d}}$ does not influence the shape of the $I-V$ characteristics because it is empty.

An increase in the applied voltage leads to changes in the behavior of $I-V$ characteristics of unloaded polymer. As is seen in insert in Fig. 2, the best linear fit for the current-voltage dependence in the high voltage range can be plotted in terms of the

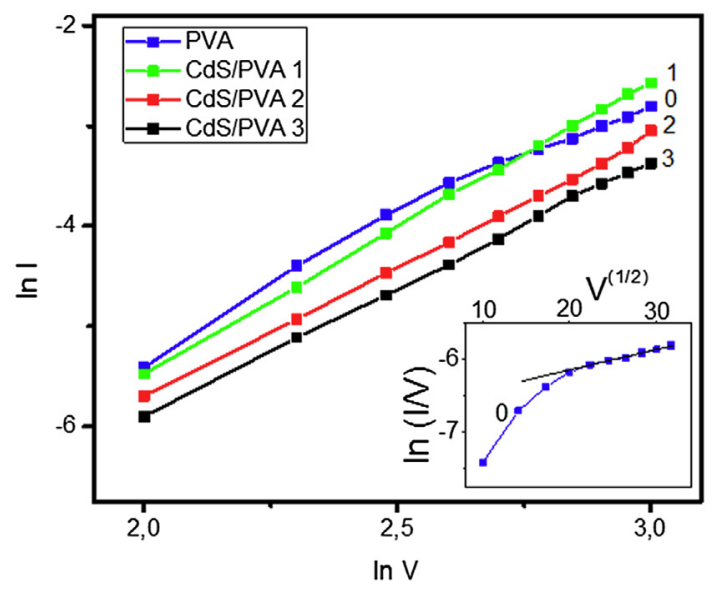

Fig. 2. Current-voltage characteristics of PVA and CdS/PVA samples in the double logarithmic coordinates. Curves: 0 - unloaded PVA, 1, 2, 3 - nanocomposites with the NPs concentrations $c_{0} / 4,2-c_{0} / 2$ and $c_{0}$, respectively. $c_{0}=4^{*} 10^{16} \mathrm{~cm}^{-3}$. Inset: the current-voltage characteristics of unloaded PVA in Poole-Frenkel coordinates.
Poole-Frenkel coordinates. Such change in conductivity behavior implies that at high fields certain donor states start to play the dominating role in conductivity. These donor states are denoted as $E_{\mathrm{d}}$ in Fig. 3a, b. While at low fields these donor states are empty, at higher fields they become filled or partly filled due to the increased injection of electrons. In other words, with an increase in the applied field, the Fermi level moves up and eventually reaches $E_{\mathrm{d}}$ (see the shaded area within $E_{\mathrm{d}}$ in Fig. $3 \mathrm{~b}$ ). The filling of the donor states changes the conductivity regime in the polymer: the increase in conductivity with voltage is determined by the lowering of the Coulomb barrier of a donor center; and, hence, the $I-V$ characteristic is described by the Poole-Frenkel relation

$I=e \cdot \mu \cdot n_{0} \cdot E \cdot \exp \cdot\left(\beta \cdot E^{1 / 2} / r \cdot k \cdot T\right)$,

where: $e$ is the elementary electron charge, $\mu$ is the charge mobility, $n_{0}$ is the concentration of charges at zero field, $\beta$ is the Poole-Frenkel constant $\left(\beta=\left(e^{3} / \pi \varepsilon \varepsilon_{0}\right)\right), k$ is the Boltzmann constant, and $r$ is the parameter describing the exponential distribution of traps within the band gap. Similar behavior of the polymer was observed for pure PVA in the high fields region in the papers $[2,4]$.

The scheme of the electronic energy states in the CdS/PVA composite at zero and low fields (Fig. 3c) differs from the corresponding scheme of unloaded PVA by the presence of deep trap states introduced into the band gap of the polymer by NPs (denoted as $E_{\mathrm{NP}}$ in Fig. 3c, d). Thus, in the presence of NPs, the total amount of states that are to be filled by injected electrons is larger than in pure

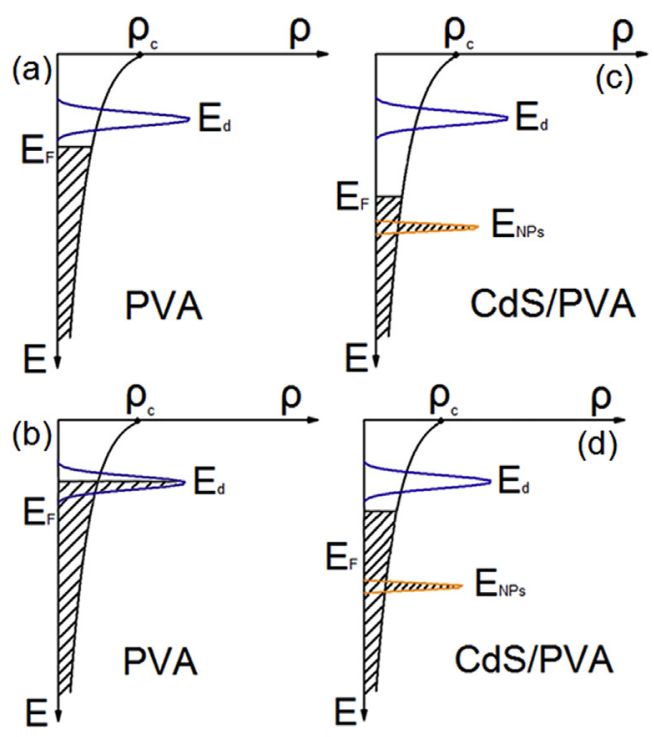

Fig. 3. Schematic diagrams of electronic energy states in the polymer (a, b) and composite (c, d). (a, c) - the case of zero and low applied field, (b, d) - the case of high field. $\rho$ - density of states; $E$ - energy counted from the bottom of the conduction band; $E_{\mathrm{d}}$ - energy of intrinsic donors of PVA; $E_{\mathrm{NPs}}$ - energy of the traps related to NPs; $E_{\mathrm{F}}-$ Fermi level; shaded areas correspond to the states filled with electrons. 
PVA. Consequently, the Fermi level in the composite at any voltage lies lower than the Fermi level in the unloaded PVA at the same voltage (Fig. 3c, d). Therefore the Fermi level in the composite would reach donor states at higher values of voltages than in the case of pure PVA. As we do not observe any changes in the slope of the conductivity regime on the $I-V$ characteristics for any composite sample (Fig. 2, curves 1-3), i.e. we do not observe the change of the conductivity regime, one can conclude that, within the whole range of applied voltages under investigation, the filling of donors with electrons injected into composite from electrodes is not achieved.

\subsection{Activation energy of conductivity}

In view of the observed linearity of the dependences $\ln \sigma$ vs. $1000 / T$ in certain temperature ranges for all samples, the concept of the activation energy of conductivity, $E_{\mathrm{a}}$, was used. The applicability of this concept to the dielectrics with exponential distribution of traps has been shown in Ref. [22]. Table 1 shows that the value of $E_{\mathrm{a}}=0.32 \mathrm{eV}$ is observed both for pure PVA and for CdS/PVA nanocomposite samples. This value correlates with the values $0.3 \mathrm{eV}$ and $0.33 \mathrm{eV}$ obtained from the dc conductivity measurements of pure PVA in similar temperature ranges [2 and 1, respectively] as well as the value $E_{\mathrm{a}}=0.3 \mathrm{eV}$ obtained from measurements of the temperature dependence of ac dielectric losses [23]. Thus, we suggest that the activation energy of $0.32 \mathrm{eV}$ is the intrinsic value of PVA and is related to the activation of electrons trapped within the density of states tail. However, neither our data nor the results of $[1,2,23]$ agree with the value $-E_{\mathrm{a}}=0.44-0.55 \mathrm{eV}$ that was observed in paper [15].

For nanocomposite samples, the curves $\ln \sigma$ vs 1000/T exhibit two slopes. One of the slope values is identical to the $E_{\mathrm{a}}=0.32 \mathrm{eV}$ activation energy that was observed for unloaded PVA; therefore, we ascribe it to a contribution from the polymeric matrix. Consequently, the second activation energy value, $E_{\mathrm{a}}=0.87 \mathrm{eV}$, is a characteristic of NPs themselves (labeled $E_{\mathrm{NPs}}$ in Fig. 3c,d). This statement is supported by the fact that this activation energy does not depend on the mass content of CdS in the nanocomposite samples. The observed value of the activation energy is smaller than the depth of the potential well formed by nano-CdS in PVA. Thus, one can assume that, with an increase in temperature, trapped electrons are ejected from some states related to the CdS/PVA interface rather than from the core of NPs (while the processes involving the release of carriers from energy levels within NPs should be seen at higher temperatures).

\subsection{Temperature coefficient of resistivity $\left(\alpha_{T}\left(K^{-1}\right)\right)$}

The observed increase of $\alpha_{\mathrm{T}}$ after the addition of NPs to the polymer can also be ascribed to the trapping of carriers by NPs. The values of $\alpha_{\mathrm{T}}$ obtained for nano-CdS/PVA composites which can be as high as $\sim 0.11$ (for the sample 3 ) are higher than the typical $\alpha_{\mathrm{T}}$ values for semiconductor thermistors $\left(0.04 \mathrm{~K}^{-1}\right)$. These values are comparable with the value $\alpha_{\mathrm{T}}=0.15 \mathrm{~K}^{-1}$ reported for nanocomposite in Ref. [20]. In that paper, composites containing CdS NPs of similar size $(5 \mathrm{~nm})$ were studied. The authors ascribed the high temperature sensitivity of the composite to the percolation regime of conductivity; the reason to assume that percolation was a dominating current mechanism in their samples was the high content - as high as $31 \mathrm{wt} \%$ - of semiconductor phase. The percolation mechanism, however, is not valid for the description of conductivity in our samples, because the content of NPs in our samples does not exceed 0.32 wt\% (note, that according to Ref. [17], a percolation threshold of PVA-CdS composite is about $13 \mathrm{wt} \%$ ). Thus, we can conclude that the domination of the percolation regime is not crucial to observation of high values of $\alpha_{\mathrm{T}}$ in the nanocomposites studied.
Due to their high $\alpha_{\mathrm{T}}$ value, nano-CdS/PVA composites are promising materials for highly-sensitive thermistors in a temperature range that is important for medical and biological studies (e.g., the human body temperature range of $35-40{ }^{\circ} \mathrm{C}$ ). In nanocomposite samples, the higher $\alpha_{\mathrm{T}}$ value (i.e., higher temperature sensitivity) corresponds to the temperature range where higher activation energy is observed (Fig. 1b). Crossover temperatures from low to high sensitivity for the samples studied here lie within the range of $30-45^{\circ} \mathrm{C}$. By adjusting the NPs concentration one can obtain material with the intended crossover temperature. Thus, sensors with two temperature ranges of sensitivity can be designed: low-sensitivity range (for temperature monitoring) and highsensitivity range (for precise temperature measurements).

Taking into account that the solid nanocomposite films studied were fabricated by the simple drying of colloidal solutions, the technologies for producing prospective nanocomposite-based thermistors could be versatile and cheap. Thermistors could be fabricated, for example, using modern printing technologies, an attractive technique for large-scale commercial production. Another possibility to use nanocomposite materials could be piece fabrication of thermistors in situ in hard-to-reach places.

\section{Conclusions}

Current-voltage characteristics and temperature dependencies of conductivity of nano-CdS/PVA composites with different fillings by semiconductor nanoparticles are studied. Current-voltage characteristics of composites exhibit superlinear (non-Ohmic) behavior. The slope in the double logarithmic scale varies from 2.9 to 2.5 as the semiconductor phase content is increased. The observed activation energies of conductivity do not depend on the concentration of NPs in the polymer and are equal to $0.32 \mathrm{eV}$ and $0.87 \mathrm{eV}$. The former value is ascribed to the PVA matrix, while the latter describes the traps related to NPs. It is shown that the crossover temperature of $\alpha_{\mathrm{T}}$ can be tuned by adjusting the NPs concentration in the composite.

\section{References}

[1] A.B. Mansour, J. Appl. Polym. Sci. 91 (2004) 3167-3173.

[2] C.U. Devi, A.K. Sharma, V.V.R.N. Rao, Mater. Lett. 56 (2002) 167-174.

[3] R.I. Mohamed, J. Phys. Chem. Solids 61 (2000) 1357-1361.

[4] N. Nagaraj, Ch.V. Subba Reddy, A.K. Sharma, V.V.R.N. Rao, J. Power Sources 112 (2002) 326-330

[5] S.C. Tjong, Y.-W. Mai, Woodhead Publ. Ser. Compos. Sci. Eng. 31 (2010) 952.

[6] T. Hanemann, D.V. Szabo, Materials 3 (2010) 3468-3517.

[7] X.C. Wu, A.M. Bittner, K. Kern, Adv. Mater. 16 (2004) 413-417.

[8] G. Kickelbick, Hybrid Materials. Synthesis, Characterization, and Applications, WILEY-VCH Verlag GmbH \& Co. KGaA, Weinheim, Germany, 2007.

[9] P.K. Khanna, Mater. Chem. Phys. 94 (2005) 454-459.

[10] H. Wang, Appl. Surf. Sci. 253 (2007) 8495-8499.

[11] M. Pattabi, B.S. Amma, K. Manzoor, Mater. Res. Bull. 42 (2007) 828-835.

[12] I.S. Elashmawi, Mater. Chem. Phys. 115 (2009) 132-135.

[13] T.G. Abdel-Malik, R.M. Abdel-Latif, A. Sawaby, S.M. Ahmed, J. Appl. Sci. Res. 4 (2008) 331-336.

[14] S. Mahendia, A.K. Tomar, S. Kumar, Mater. Sci. Eng. B 176 (2011) 530-534.

[15] S. Kulanthaisami, D. Mangalaraj, S.K. Narayandass, Eur. Polym. J. 3 (1995) 969-975.

[16] A. Lewandowski, K. Skorupska, J. Malinska, Solid State Ionics 133 (2000) $265-271$.

[17] F. El-Tantawy, K.M. Abdel-Kader, F. Kaneko, Y.K. Sung, Eur. Polym. J. 40 (2004) 415-430.

[18] A.E. Varfolomeev, A.V. Volkov, D.F. Zaretsky, M.A. Moskvina, V.Z. Mordkovych, J. Solid State Lett. 30 (2004) 1-6.

[19] G. Yu. Rudko, A.O. Kovalchuk, V.I. Fediv, Q. Ren, W.M. Chen, I.A. Buyanova, G. Pozina, Thin Solid Films 543 (2013) 11-15.

[20] K. Lakshminarayana, Y. Dasaradhudu, V.V.R.N. Rao, Mater. Lett. 21 (1994) 425-430.

[21] A. Rose, Phys. Rev. 97 (1955) 1531-1537.

[22] V. Kumar, S.C. Jain, A.K. Kapoor, J. Poortmans, R. Mertens, J. Appl. Phys. 94 (2003) 1283-1285.

[23] I.Yu. Prosanov, N.F. Uvarov, Solid State Phys. 54 (2012) 393-396. 